\title{
Are There Any Differences in Sleep Disorder, Quality of Life and Gastric Motility Among Subtypes of Functional Dyspepsia?
}

\author{
Seon-Young Park* and Jong-Sun Rew \\ Department of Internal Medicine, Chonnam National University Hosptial, Gwangju, Korea
}

Article: Impact of sleep disorders, quality of life and gastric emptying in distinct subtypes of functional dyspepsia in Japan Yamawaki H, Futagami S, Shimpuku M, et al

(J Neurogastroenterol Motil 2014;20:104-112)

The pathophysiology of functional dyspepsia (FD) is related to symptoms characterized by multifunctional disorders of the upper gastrointestinal (GI) tract, such as disorders of GI motility, abnormal acid secretion, visceral hypersensitivity, Helicobacter $p y$ lori infection and potent psychological factors. FD has been subclassifed into 2 disease categories under the Rome III classification criteria: epigastric pain syndrome (EPS) and postprandial distress syndrome (PDS). ${ }^{1}$ We read with interest the original article by Yamawaki et $\mathrm{al}^{2}$ regarding the prevalence and relationship for sleep disorders and quality of life in patients with subtypes of FD (EPS, PDS and EPS-PDS overlap).

Sleep disorder is a common medical problem, and has been associated with functional GI disorders including gastroesophageal reflux disease and irritable bowel syndrome. ${ }^{3,4}$ The higher prevalence of sleep disorder in FD patients has been reported by previous other studies. In this study, the authors reported that sleep disorders distribution in 3 FD subtypes was significantly higher compared to that in healthy volunteers. However, the study did not suggest whether treatment for FD improved the quality of sleep. Actually, it is possible that both sleep disorders and functional GI disturbances may be the result of some underlying problem, such as depression, anxiety or other psychological conditions. Geeraerts et $\mathrm{al}^{5}$ have reported that experimentally induced anxiety was associated with decreased gastric compliance and accommodation to a meal and with increased epigastric symptoms score during a standard nutrient challenge in health volunteers. Some studies have suggested that the presence of anxiety could modulate the gut function and produce GI disorders. ${ }^{6}$ So, previous study showed a positive correlation with state anxiety. ${ }^{7}$ However, this study did not suggest that correlation between the gastric emptying (Tmax) and State-Trait Anxiety Inventory score (STAI-trait/-state score). Instead, this study showed that there was a significant relationship between total Glasgow dyspepsia severity score (GDSS) and global Pittsburgh Sleep Quality Index (PSQI) score in PDS and EPS. And also, the study showed that health-related quality of life by measuring 8-item short form health survey (SF-8) was significantly associated with sleep disorder (global PSQI) in FD

Received: November 29, 2013 Revised: None Accepted: December 2, 2013

(c) This is an Open Access article distributed under the terms of the Creative Commons Attribution Non-Commercial License (http://creativecommons. org/licenses/by-nc/3.0) which permits unrestricted non-commercial use, distribution, and reproduction in any medium, provided the original work is properly cited.

*Correspondence: Seon-Young Park, MD

Department of Internal Medicine, Chonnam National University Medical School, 42, Jebong-ro, Dong-ku, Gwangju 501-757, Korea Tel: +82-62-220-6296, Fax: +82-62-228-1330, E-mail: drpsy@naver.com

Financial support: None.

Conflicts of interest: None. 
subtypes patients.

In a previous study, they reported that Tmax value as a marker of gastric emptying in PDS patients was significantly greater compared with healthy volunteers. ${ }^{8,9}$ The study showed that there were significant differences between 3 subtypes of FD patients and health volunteers. However, the study did not suggest a difference among 3 subtypes of FD patients.

Despite several limitations such as small number of patients and lack of data about the FD treatment on quality of sleep, it provides us about prevalence for sleep disorders, gastric motility and quality of life in each subtype of FD patients. Further studies are needed to evaluate the physiologic factors and psychological factors in each FD subtype. And, it is necessary to clarify that treatment for each subtype of FD patients for alleviating the dyspeptic symptoms may improve the quality of life or quality of sleep.

\section{References}

1. Tack J, Talley NJ, Camilleri M, et al. Functional gastroduodenal disorders. Gastroenterology 2006;130:1466-1479.

2. Yamawaki H, Futagami S, Shimpuku M, et al. Impact of sleep disorders, quality of life and gastric emptying in distinct subtypes of func- tional dyspepsia in Japan. J Neurogastroenterol Motil 2014;20:104112 .

3. Fass R, Fullerton S, Tung S, Mayer EA. Sleep disturbances in clinic patients with functional bowel disorders. Am J Gastroenterol 2000; 95:1195-2000

4. Jarrett M, Heitkemper M, Cain KC, Burr RL, Hertig V. Sleep disturbance influences gastrointestinal symptoms in women with irritable bowel syndrome. Dig Dis Sci 2000;45:952-959.

5. Geeraerts B, Vandenberghe J, Van Oudenhove L, et al. Influence of experimentally induced anxiety on gastric sensorimotor function in humans. Gastroenterology 2005;129:1437-1444.

6. Lorena SL, Tinois E, Brunetto SQ, Camargo EE, Mesquita MA. Gastric emptying and intragastric distribution of a solid meal in functional dyspepsia: influence of gender and anxiety. J Clin Gastroenterol 2004;38:230-236.

7. De la Roca-Chiapas JM, Solis-Ortiz S, Fajardo-Araujo M, Sosa M, Córdova-Fraga-Fraga T, Rosa-Zarate A. Stress profile, coping style, anxiety, depression, and gastric emptying as predictors of functional dyspepsia: a case-control study. J Psychosom Res 2010;68:73-81.

8. Shindo T, Futagami S, Hiratsuka T, et al. Comparison of gastric emptying and plasma ghrelin levels in patients with functional dyspepsia and non-erosive reflux disease. Digestion 2009;79:65-72.

9. Futagami $\mathrm{S}$, Yamawaki $\mathrm{H}$, Izumi $\mathrm{N}$, et al. Impact of sleep disorders in Japanese patients with functional dyspepsia (FD): nizatidine improves clinical symptoms, gastric emptying and sleep disorders in FD patients. J Gastroenterol Hepatol 2013;28:1314-1320. 